\title{
Fetal Cardiac Interventricular Septal Thickness at 28-37 Weeks of Gestation in Nepalese Population
}

\author{
Kafle $\mathbf{P}^{1}$, Ansari MA ${ }^{1}$, Khanal $\mathbf{U}^{1}$ \\ ${ }^{1}$ Department of radiology and Imaging, Institute of Medicine, Tribhuvan University Teaching \\ Hospital, Kathmandu, Nepal
}

\begin{abstract}
Objective: The purpose of this study is to establish a new reference range for fetal interventricular septal thickness in uncomplicated pregnancy at 28-37 weeks of gestation. Materials and Methodology: This was a prospective cross sectional study involving 300 singleton pregnancies between 28-37 weeks of gestation without any known risk factors of adverse pregnancy outcome who were referred for routine obstetric examination. The protocol included the prenatal 2-dimensional M-mode echocardiographic measurements of fetal IVST and data were used to construct the normograms and percentile fitted curves for different gestational age. The relationship between the IVSD and IVSS and gestational age were determined. Results: A total of 300 measurements were obtained. The normal values of the IVSD and IVSS according to gestational age were presented as $5^{\text {th }}, 50^{\text {th }}$ and $95^{\text {th }}$ percentile ranks. The correlation coefficients ( $r$ ) between the IVSD and IVSS and gestational age were 0.19 and 0.14 , respectively. The weak correlation may be probably due to small sample size. The IVSD and IVSS were not statistically different with advancing gestation. The $95^{\text {th }}$ percentile of the IVSD was 4.57 millimeters $(\mathrm{mm})$ (range $=4.12$ to $4.62 \mathrm{~mm}$ ) and IVSS was $6.67 \mathrm{~mm}$ (range $=5.81$ to $6.77 \mathrm{~mm}$ ). Conclusion: The normal values of fetal IVSD and IVSS in a Nepalese population from 28 to 37 weeks' gestation were established. This could be used as a baseline data in detecting the asymmetrical septal hypertrophy during fetal life.
\end{abstract}

Keywords: Fetal echocardiography, Interventricular septal thickness, Maternal Diabetes Mellitus.

\section{Introduction}

Congenital heart disease (CHD) occurs in approximately $0.8 \%$ of all live-born humans. ${ }^{1}$ The causes are multifactorial and include genetic as well as environmental (in utero) factors. Maternal diabetes mellitus
(MDM) is known to increase the likelihood

Correspondence to: Dr. Pankaj Kafle, Department of Radiodiagnosis and Imaging, Institute of Medicine, Tribhuvan University Teaching Hospital, Kathmandu, Nepal

Email Id: pankajkafle@gmail.com 
of fetal CHD, with an estimated increased risk of up to $8.5 \%$ of live births. ${ }^{2}$ Most types of cardiac structural lesions have been associated with MDM, ranging from small septal defects to duct-dependent heart disease. ${ }^{3-6}$ As a separate concern, there is an increased risk for the development of hypertrophic cardiomyopathy in the third trimester in this population.

In particular, cardiomyopathy, characterized by a thick interventricular septum, is often seen in offspring's of women with insulindependent diabetes. ${ }^{7,8}$ Characteristically, the interventricular septa in these infants are disproportionately thickened for gestational age and are disproportionately large in relation to the left ventricular posterior wall dimension. ${ }^{9}$ These changes were seen in association with impaired fetal diastolic function and it might be one of the leading causes of perinatal morbidity and mortality in pregnancies complicated by type 1 diabetes. ${ }^{8,9}$

Prenatal detection of the asymmetrical septal hypertrophy in the third trimester might have clinical relevance as persistent pulmonary hypertension of the newborn and idiopathic respiratory distress syndrome have been reported in infants of diabetic mothers with this condition. To date, however, only a little information is available on the knowledge about fetal cardiovascular dimensions to permit developmental methods of quantification that can help to describe these changes. There are several normal reference ranges of fetal IVST currently in use. However, no such reference ranges are published for the Nepalese population.

The purpose of prenatal cardiac screening is to identify those patients with defects that may require further evaluation and treatment, and to provide appropriate counseling to the family in a timely manner. ECHO, therefore, has become a fundamental tool for intrauterine cardiologic diagnosis. ${ }^{10}$ The immediate implication is that many heart diseases and arrhythmias might be treated intrauterus and, in severe cases needing immediate post-partum emergency clinicalsurgical management, early diagnosis allows anticipated planning. ${ }^{11}$

These data could provide the threshold values, serving as a means for prenatal evaluation in fetuses of diabetic pregnancies in detecting the asymmetrical septal hypertrophy during fetal life.

\section{Materials and Methodology}

This was a prospective cross sectional study involving 300 singleton pregnancies between 28 and 37 weeks of gestation without any known risk factors of adverse pregnancy outcome who were referred for routine obstetric examination. The protocol included the M-mode echocardiographic measurements of interventricular septal thickness during systole and diastole of cardiac cycle at four chamber view of heart and data were used to establish the normograms and percentile fitted curves of IVST for different gestational age.

\section{Interventricular septal thickness measurement by M-mode Echocardiography}

During the examination, the mother reclined supinely, with some rotation either to the right or left side to facilitate the evaluation as described elsewhere. The M-mode cursor was placed perpendicular to the 


\section{IVST RECORDING}

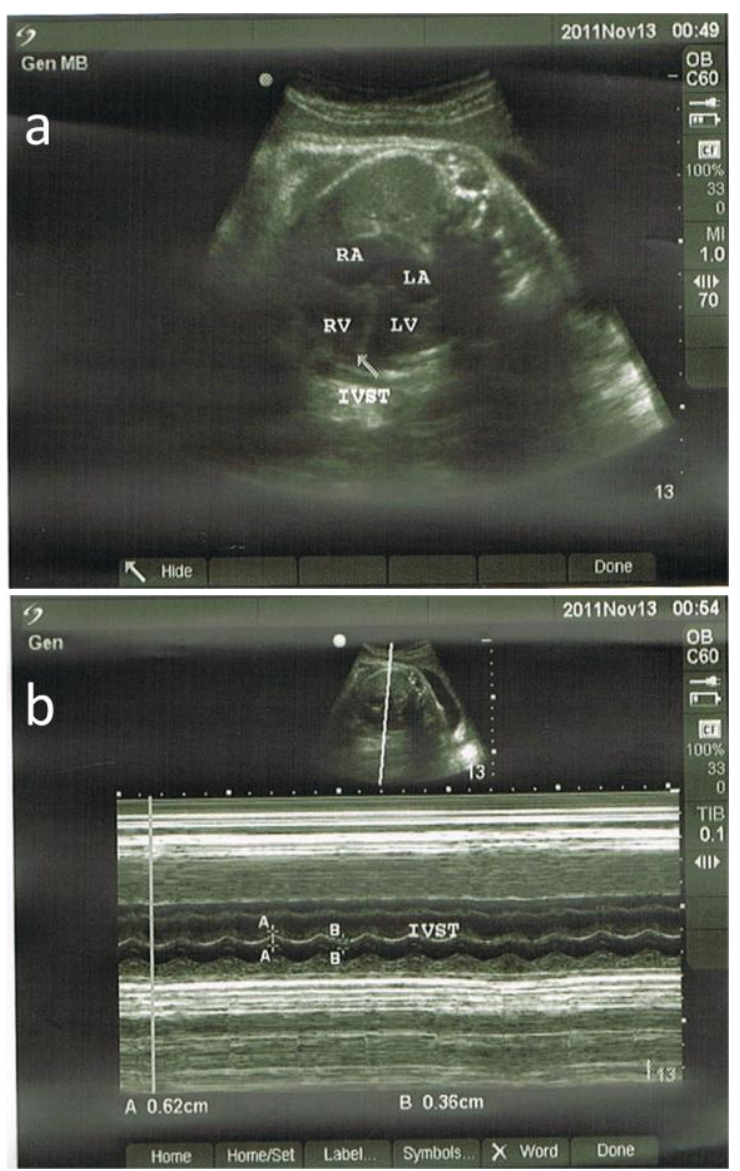

The interventricular septal thickness: (a) 4-chamber view (b) Interventricular septum by M-mode echocardiography. The measurement of Interventricular septal thickness during diastole and systole: the distance between the outer edges of each margin was measured in millimeter. (IVST: interventricular septum; A-A: interventricular septal thickness during systole; B-B: interventricular septal thickness during diastole; LA: left atrium; LV: left ventricle; $R A$ : right atrium; $R V$ : right ventricle).

interventricular septum just below the atrioventricular valves at the level of the 4chamber view according to the standards recommended by the American Society of Echocardiography. A biventricular activity tracing was used to measure the IVST with electronic calipers. The IVST was measured during fetal apnea. It was taken on the distance between the outer edges of each margin and was measured in millimeter $(\mathrm{mm})$. The present study was performed in the usual manner, usually brief, normally lasting about or less than 15 minutes with careful attention given to gain a setting adjusted to produce the best possible image.

\section{Statistical analysis}

The statistical analysis was carried out by using SPSS software package version 17.0 (SPSS Inc., Chicago, IL, USA). Obstetric characteristics are presented as mean + standard deviation. The IVSD and IVSS obtained from the fetuses were plotted against gestational age and the correlation coefficients were determined by using Pearson's correlation. The normal values of the IVSD and IVSS were presented as 5th, 50th and 95th percentile ranks. P-value < 0.05 was considered statistically significant. Normograms and percentile fitted curves were obtained.

It was found that the standard deviation of fetal IVST was approximately $1.23 \mathrm{~mm}$ for IVSD and $1.61 \mathrm{~mm}$ for IVSS.

\section{Observations and Results}

The median for higher gestational ages were significantly higher than lower ones both for IVSS (chi square $=28.22, p=0.001$ ) and IVSD (chi square $=35.56, \mathrm{p}<0.001$ ). The median for IVSS was 4.1 and for IVSD were 2.9.

The IVSD and IVSS were not statistically different with advancing gestation from 28 to 37 weeks gestation $(p<0.05)$. The 95 th 
The 5 th, $50^{\text {th }}$ and 95 th percentiles of the IVSD and IVSS over gestational age are shown.

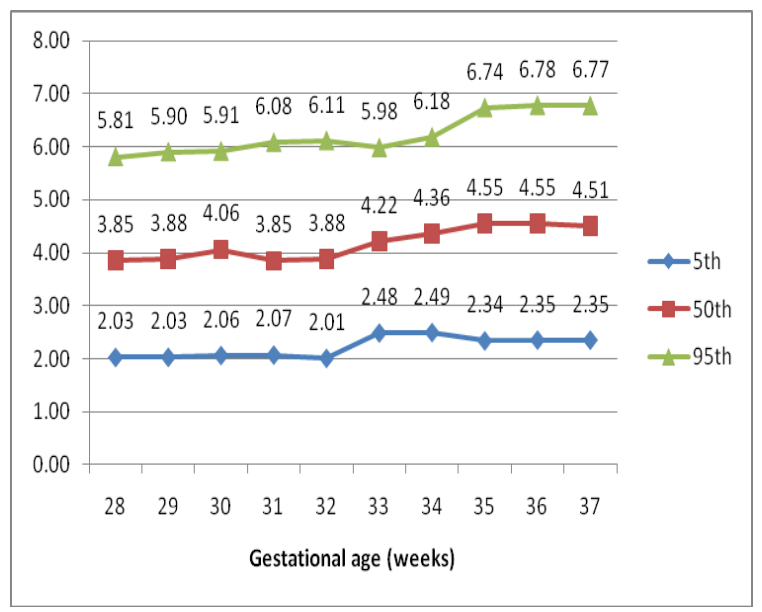

The $5^{\text {th }}, 50^{\text {th }}$ and $95^{\text {th }}$ percentile value of IVSS in relation to the gestational age.

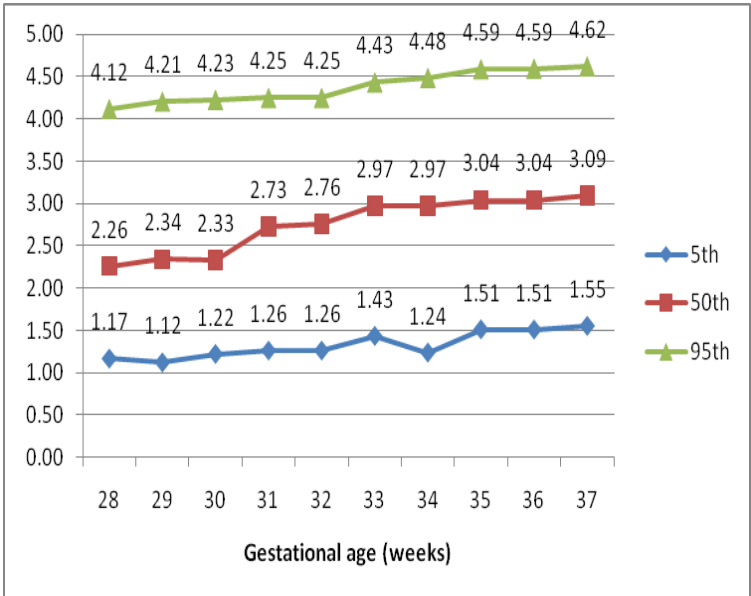

The $5^{\text {th }}, 50^{\text {th }}$ and $95^{\text {th }}$ percentile value of IVSD in relation to the gestational age.

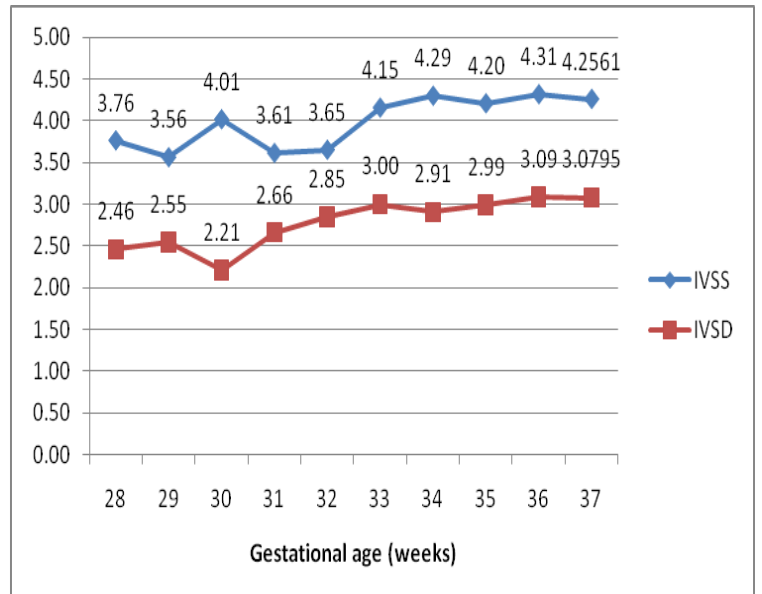

percentile of the IVSD and IVSS were 4.57 $\mathrm{mm}$ (range $=4.12$ to $4.62 \mathrm{~mm}$ ) and $6.67 \mathrm{~mm}$ (range $=5.81$ to $6.77 \mathrm{~mm}$ ), respectively.

\section{Discussion}

Detailed fetal anatomic surveys in the early second trimester are common practice and typically include examination of both fourchamber and outflow tract views of the fetal heart. In present study, cross-sectional observations were obtained in 300 uncomplicated singleton pregnancies between 28 to 37 weeks of gestational age. Reference ranges for interventricular septal thickness of fetal heart was established based on these observations.

Ultrasound imaging of the normal fetal heart was initially described in 1972 by Winsberg. ${ }^{12}$ It was nearly a decade later when others began to document normal growth parameters of the fetal heart ${ }^{13-16}$ and the prenatal diagnosis of the cardiac pathology.

Cooper et al. ${ }^{17}$ had documented that for the population of infants found to have asymmetrical septal hypertrophy at birth, the thickened interventricular septum was usually demonstrated at 31 to 34 weeks gestation. At this point in gestation, the fetuses showed a significant increase in the Interventricular Septal thickness (IVST) compared to those who were not proved to have asymmetrical septal hypertrophy at birth. $^{18}$

Gandhi et al. ${ }^{19}$ reported that the IVST was greater in the fetuses of insulin- requiring diabetic mothers than the normal fetuses of non-diabetic mothers between 32 to 36 weeks gestation. According to gestational 
age group studies among the normal fetuses and the fetuses of diabetic mothers, it was shown that the IVST were significantly greater in the group of 32 to 36 weeks' gestation than those in the group of 20 to 24 and 28 to 31 weeks gestation.

Previous studies have reported that the fetal IVST during both diastole and systole increased significantly in a linear fashion with advancing gestation from 18 weeks to term. $^{20-23}$

Tan et al. ${ }^{21}$ showed that the $95^{\text {th }}$ percentile of the IVSD among 100 normal fetuses from 32 to 35 weeks gestation was approximately $4 \mathrm{~mm}$, whereas Veille et al. ${ }^{22}$ reported that these values of the IVSD and IVSS were approximately $3.0 \mathrm{~mm}$ and $4.5 \mathrm{~mm}$, respectively. Zielinsky ${ }^{10}$ found that the asymmetrical septal hypertrophy among the fetuses of diabetic mothers had the mean IVST of $7.12+1.6 \mathrm{~mm}$ at the mean gestational age of 32 weeks.

In the present study, there was little or no relationship between the fetal IVST and gestational age. The values of fetal IVST were not significantly different with advancing gestation from 28 to 37 weeks. The 95th percentile of the IVSD and IVSS were $4.57 \mathrm{~mm}$ and $6.67 \mathrm{~mm}$, respectively. These findings were not similar to the previous studies ${ }^{20-23}$ which reported that the mean septal size during both diastole and systole were increased significantly in a linear fashion with advancing gestation from 18 weeks to term.

Muller et $\mathrm{al}^{24}$ reviewed routine fetal echocardiographic screening in all high-risk patients (including those with MDM) with normal obstetric four-chamber and outflow tract views. Only two cases of major CHD were identified that had not been seen on prior obstetric scans, and no cases were found among patients with isolated preexisting diabetes mellitus.

Odibo et al. ${ }^{25}$ specifically addressed the costeffectiveness of screening all pregnant diabetic women and suggested that selective screening of diabetic mothers with abnormal obstetric anatomical surveys is more costeffective than is universal screening of all diabetic mothers. $^{26}$

The result obtained in the present study, showing poor correlation does matches with the similar study conducted on a Thai population on 32-35 weeks of gestational age. $^{27}$

\section{Conclusion}

A total of 300 uncomplicated singleton pregnancies between 28 and 37 weeks of gestation were included in this cross sectional study. Reference ranges for Interventricular septal thickness of fetal heart were established.

Minimal linear increment in the IVST with advancing gestational age was obtained. The present study could be used as a baseline data for evaluation of the fetus at risk for hypertrophic cardiomyopathy, especially in the light of recent epidemiologic data linking increased incidence of diabetes in the general population.

\section{Acknowledgement}

The authors wish to thanks Department of Obstetrics and Gynecology of Marajgunj 
medical campus without which the completion of this project would have vail.

1. Mitchell SC, Korones SB, Berendes HW. Congenital heart disease in 56,109 births. Incidence and natural history. Circulation 1971; 43: 323-332.

2. Becerra JE, Khoury MJ, Cordero JF, Erickson JD. Diabetes mellitus during pregnancy and the risks for specific birth defects: a population-based case-control study. Pediatrics 1990; 85:1 9.

3. Cooper MJ, Enderlein MA, Dyson DC, Roge CL, Tarnoff H. Fetal echocardiography: retrospective review of clinical experience and an evaluation of indications. Obstet Gynecol 1995; 86: 577-582.

4. Ferencz C, Rubin JD, McCarter RJ, Clark EB. Maternal diabetes and cardiovascular malformations: predominance of double outlet right ventricle and truncus arteriosus. Teratology 1990; 41: 319-326.

5. Hamar BD, Dziura J, Friedman A, Kleinman CS, Copel JA. Trends in fetal echocardiography and implications for clinical practice: 1985 to 2003. J Ultrasound Med 2006; 25: 197-202.

6. Kitzmiller JL, Gavin LA, Gin GD, Jovanovic-Peterson L, Main EK, Zigrang WD. Preconception care of diabetes. Glycemic control prevents congenital anomalies. JAMA 1991; 265: 731-736.

7. Pildes RS. Infants of diabetic mothers. N Engl J Med 1973; 289: 902-4.
8. Mace S, Hirschfeld SS, Riggs T, Fanaroff AA, Merkatz IR, Franklin W. Echocardiographic abnormalities in infants of diabetic mothers. J Pediatr 1979; 95: 1013-9.

9. Rizzo G, Arduini D, Romanini C. Cardiac function in fetuses of type I diabetic mothers. Am J Obstet Gynecol 1991; 164: 837-43.

10. Zielinsky P. Role of prenatal echocardiography in the study of hypertrophic cardiomyopathy in the fetus. Echocardiography 1991; 8: 661- 8.

11. Bromley B, Estroff JA, Sanders SP, Parad R, Roberts D, Frigoletto FD Jr, et al. Fetal echocardiography: accuracy and limitations in a population at high and low risk for heart defects. Am J Obstet Gynecol. 1992; 166(5):1473-81.

12. Winsberg F: Echocardiography of fetal newborn heart. Invest Radiol 1972; 7:152-158.

13. Allan, LD, Tynan MJ, Campbell S, et al: Echocardiography and anatomical correlates in the fetus. Br Heart J 1980; 44:444-447.

14. Lange LW, Sahn DJ, Allen HD, et al: Qualitative real-time cross sectional echocardiographic imaging of the human fetus during second half of the pregnancy. Circulation 1980; 62:799805.

15. Sahn DJ, Lange LW, Allen HD, et al: Qualitative real-time cross sectional echocardiography in the developing normal human fetus and newborn. Circulation 1980; 62:588-603. 
16. Huhta JC, Hagler DJ, Hill LM: Two Dimensional echocardiography assessment of normal fetal cardiac anatomy. J Reprod Med 1984; 29:162165.

17. Cooper MJ, Enderlein MA, Tarnoff H, Roge CL. Asymmetric septal hypertrophy in infants of diabetic mothers. Fetal echocardiography and the impact of maternal diabetic control. Am J Dis Child 1992; 146: 226-9.

18. Allan LD, Joseph MC, Boyd EGCA, Campbell S, Tynan M. M-mode echocardiography in the developing human fetus. Br Heart J 1982; 47: 573 83.

19. Gandhi JA, Zhang XY, Maidman JE. Fetal cardiac hypertrophy and cardiac function in diabetic pregnancies. Am J Obstet Gynecol 1995; 173: 1132-6.

20. Veille JC, Hanson R, Steele L, Tatum K. M-mode echocardiographic evaluation of fetal and infant hearts: Longitudinal follow-up study from intrauterine life to year one. Am J Obstet Gynecol 1996; 175: 922-8.

21. Weber HS, Copel JA, Reece EA, Green J, Kleinman CS. Cardiac growth in fetuses of diabetic mothers with good metabolic control J Pediatr 1991; 118: 103-7.

22. Reller MD, Tsang RC, Meyer TA, Braun CP. Relationship of prospective diabetes control in pregnancy to neonatal cardiorespiratory function. J Pediatr 1985; 106: 86-90.
23. Halliday HL. Hypertrophic cardiomyopathy in infants of poorlycontrolled diabetic mothers. Arch Dis Child 1981; 56: 258-63.

24. Muller PR, James A, Feldman K, Herlong JR. Utility of fetal echocardiogram in high-risk patients. Aust N Z J Obstet Gynaecol 2005; 45: 117-121.

25. Odibo AO, Coassolo KM, Stamilio DM, Ural SH, Macones GA. Should all pregnant diabetic women undergo a fetal echocardiography? A cost-effectiveness analysis comparing four screening strategies. Prenat Diagn 2006; 26: 39-44.

26. Hendler I, Blackwell SC, Bujold E, Treadwell MC, Mittal P, Sokol RJ, Sorokin Y. Suboptimal second-trimester ultrasonographic visualization of the fetal heart in obese women: should we repeat the examination? $J$ Ultrasound Med 2005; 24: 1205-1209.

27. Lawan Patchakapat, Boonchai Uerpairojkit, Teera Wacharaprechanont, Saknan Manotaya, Somchai Tanawattanacharoen, Dhiraphongs Charoenvidhya. Interventricular Septal Thickness of Thai Fetuses at 32 to 35 Weeks Gestation. J Med Assoc Thai 2006; 89 (6):748-54. 